\title{
A literature review of research exploring the experiences of overseas nurses in the United Kingdom (2002-2017)
}

Review article

Yi-Qing Lin, Yun Ding", Jiong-Yan Li*

Nursing Department, Suzhou Municipal Hospital, Suzhou, Jiangsu 215000, China

Received: 18 July 2017; Accepted: 25 August 2017; Published: 20 March 2018

Abstract: Objectives: The goals of this work were to critically explore the challenges faced by overseas nurses during their working careers in the UK, to critically explore the available support for overseas nurses, and to make recommendations on how to minimize the effects of these challenges, as well as how to provide more support for overseas nurses during their working careers in the UK.

Methods: A systematic and comprehensive literature review was conducted to explore the said objectives. A search was conducted in the Cumulative Index to Nursing and Allied Health Literature (CINAHL), Scopus, and Academic Search Complete databases for relevant research. Inclusion and exclusion criteria were used to select the included articles. A total of 8 studies including 6 qualitative studies, 1 quantitative study, and 1 mixed-method study were selected for literature review. The validity and reliability of these 8 studies were analyzed by using 2 appraisal tools.

Results: This review reveals 4 challenges, which overseas nurses experience in the past 15 years, including cultural differences, communication issues, unequal opportunities, and de-skilling. These challenges have a negative impact on overseas nurses' emotions and career development. The formal and informal supports are revealed in this review. Various supports can help overseas nurses to work better.

Conclusions: This review reveals 4 challenges faced by overseas nurses, including cultural differences, communication issues, and unequal opportunities and de-skilling. These challenges naturally have a profound negative impact on the emotions and career development of overseas nurses. Additionally, the formal and informal support systems available to these nurses are reviewed. Various support systems can help overseas nurses work more effectively.

Keywords: overseas nurses $\bullet$ work experiences $\bullet$ challenges $\bullet$ supports $\bullet$ cultural differences $\bullet$ communication issues $\bullet$ de-skilling $\bullet$ unequal opportunities

(c) Shanxi Medical Periodical Press.

\section{Introduction}

\subsection{The recruitment of overseas nurses in the UK}

The migration of nurses has become a global phenomenon in the 21 st century. ${ }^{1}$ Indeed, the World Health Organization $(\mathrm{WHO})^{2}$ has highlighted the prominence of nurse migration, especially since 2000 , to the UK and the USA. In the UK, a decades-long tradition of recruiting overseas nurses to make up for the shortage of nurses has become entrenched. ${ }^{3}$ In recent years, recruiting overseas nurses has become an indispensable task of the Department of Health to alleviate staffing shortages. In 2012, the Department of Health recruited 5,000 Spanish nurses to serve within the British health-care system. It is planned to recruit more nurses from China. ${ }^{4}$ Meanwhile, the government has signed several relevant 
agreements with countries facing a surplus of nurses. For example, an agreement was made with India to import approximately 6,000 nurses to work in the UK. This was beneficial in alleviating the staffing crisis to some degree. ${ }^{4}$ The Royal College of Nursing $(\mathrm{RCN})^{5}$ claimed that there is still an estimated national shortage of 22,000 nurses. Long-term nursing shortages in the UK mean that managers must actively recruit experienced nurses from both developed and developing countries. ${ }^{5}$

However, although the government has worked on importing a number of overseas nurses for many years through active policies and strategies, the shortage of nurses has not yet been rectified, in large part due to the rapidly increasing domestic needs of caregivers. Lintern ${ }^{6}$ believed that this chronic shortage of nurses would last for several years, but he predicted that the National Health Service (NHS) is likely to have more nurses than it needs by 2016. The statistics from the Nursing Times confirmed these predictions (Table 1). In 2010, there was a balance between supply of and demand for nurses in the British health-care system. However, this balance was immediately upset in 2016, again resulting in a shortage of 47,545 nurses. The Nursing Times revealed that it is a large challenge for managers to sustain the supply and demand of the future nursing workforce, particularly when funds are limited. Additionally, it was acknowledged that the health-care service is failing to provide communities with enough care and is therefore in urgent need of nurses to fill emergency departments. ${ }^{6}$ The $\mathrm{RCN}^{7}$ also highlighted the fact that this shortage of nurses will continue in the UK and that the scale of the shortage cannot be predicted. The RCN argued that this shortage will affect not only the health of current nurses, which may lead to a high turnover rate, but also the quality of care and even patient safety.

\begin{tabular}{l|l}
\hline a) Inclusion criteria & b) Exclusion criteria \\
\hline \hline $\begin{array}{l}\text { Articles published in English } \\
\begin{array}{l}\text { Articles published within the past } \\
15 \text { years. }\end{array}\end{array}$ & $\begin{array}{l}\text { Articles in another language } \\
\text { Articles on overseas nurses who } \\
\text { work in countries other than } \\
\text { the UK }\end{array}$ \\
$\begin{array}{l}\text { Peer-reviewed journals } \\
\text { Articles focused on home nurses } \\
\text { and overseas student nurses } \\
\text { working in the UK }\end{array}$ \\
$\begin{array}{l}\text { Articles focused on all overseas } \\
\text { nurses in the UK (including } \\
\text { minority ethnic nurses, black } \\
\text { nurses, Asian nurses, registered } \\
\begin{array}{l}\text { overseas nurses, and refresher } \\
\text { overseas nurses) }\end{array}\end{array}$ & $\begin{array}{l}\text { Articles published prior to 2002 } \\
\text { Overseas nurses' former } \\
\text { experiences before arriving in } \\
\text { the UK }\end{array}$ \\
\hline
\end{tabular}

Table 1. Inclusion and exclusion criteria

\subsection{The importance of focusing on the experiences of overseas nurses}

Overseas nurses come from different countries or regions and have different beliefs and cultures. ${ }^{8}$ Statistics from the Nursing and Midwifery Council (NMC) provide information on the top 10 countries of origin (Figure 1). Over the past 10 years, the majority of overseas nurses have come from non-European Union countries. The main countries to export nurses were the Philippines, India, South Africa, and Australia. Until 2015, there were as many as 22,500 overseas nurses from the Philippines working in the UK. However, the health-care systems in those countries have many differences relative to the British health-care system due to a number of economic, social, and cultural factors. ${ }^{8}$ This means that overseas nurses may have more difficulties during their career in a new working environment. Therefore, Nichols and Campbell highlighted the importance of exploring the experiences of overseas nurses working in the current British health-care system. ${ }^{9}$

Statistics from the NMC demonstrate the proportion of foreign nurses in the UK. Although foreign nurses accounted for only approximately one-sixth of the nursing labor workforce, the population of overseas nurses in the UK is quite large, numbering 94,851 nurses (including 28,570 European Union nurses and 66,281 nonEuropean Union nurses) (Figure 2). Numerous overseas nurses are working in the UK, and their experiences are a topic of concern for the British health-care system. In addition, after reviewing several case studies, Buchan and Dovlo ${ }^{10}$ found that managers are heavily dependent on overseas nurses as a core group of the workforce in the NHS and some private sectors. Many clinical fields currently rely on overseas nurses to satisfy their staffing needs. ${ }^{9}$ Overseas nurses are becoming increasingly important in the development of the British health-care service. ${ }^{9}$ Hence, it is necessary to pay more attention to

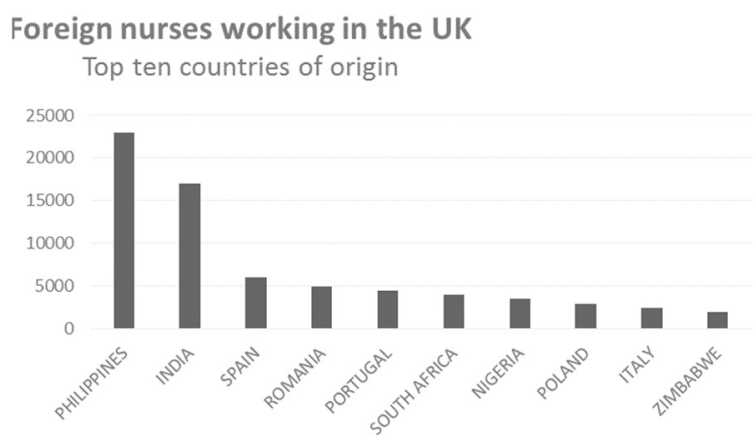

Figure 1. Foreign nurses working in the UK. Source: Nursing and Midwifery Council - Title: Recruiting foreign nurses is "frustrating and expensive" 


\section{Proportion of foreign nurses in the UK}

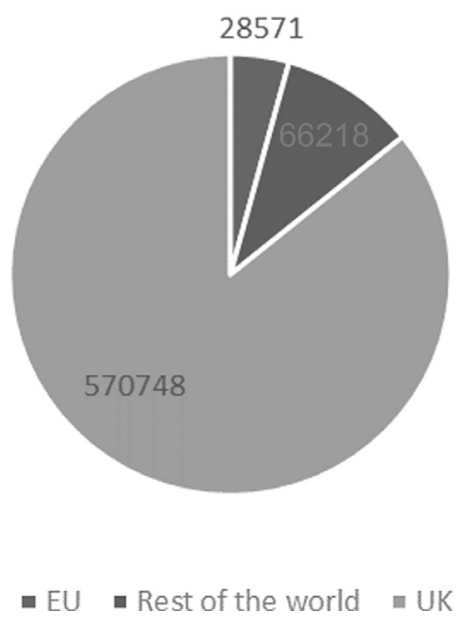

Figure 2. Proportion of foreign nurses in the UK. Source: Nursing and Midwifery Council - Title: Migrant salary rules may cost the NHS nurse jobs, the union warns their experiences, particularly given the limited supply of nursing labor. In addition, some negative experiences of overseas nurses have increased the levels of stress and burnout for numerous nurses on the frontline, and their turnover rate is higher than ever before. ${ }^{11}$ Developing a complete picture of their experiences will be beneficial for policymakers and employers, allowing them to take action in terms of retention. Furthermore, this information will help in the creation of a friendlier and more culturally diverse workforce.

Most previous research ${ }^{12,13}$ has only focused on the negative experiences of overseas nurses in the UK. However, there is little research assessing both sources of challenge and sources of support. ${ }^{14,15}$ Additionally, many studies have only studied nurses from 1 country or race. The results, therefore, cannot be generalized to all overseas nurses working in the UK. Furthermore, although Nichols and Campbell ${ }^{16}$ have produced an integrative review on this topic, they only reviewed articles written between 1995 and 2007 . With the rapid develop-

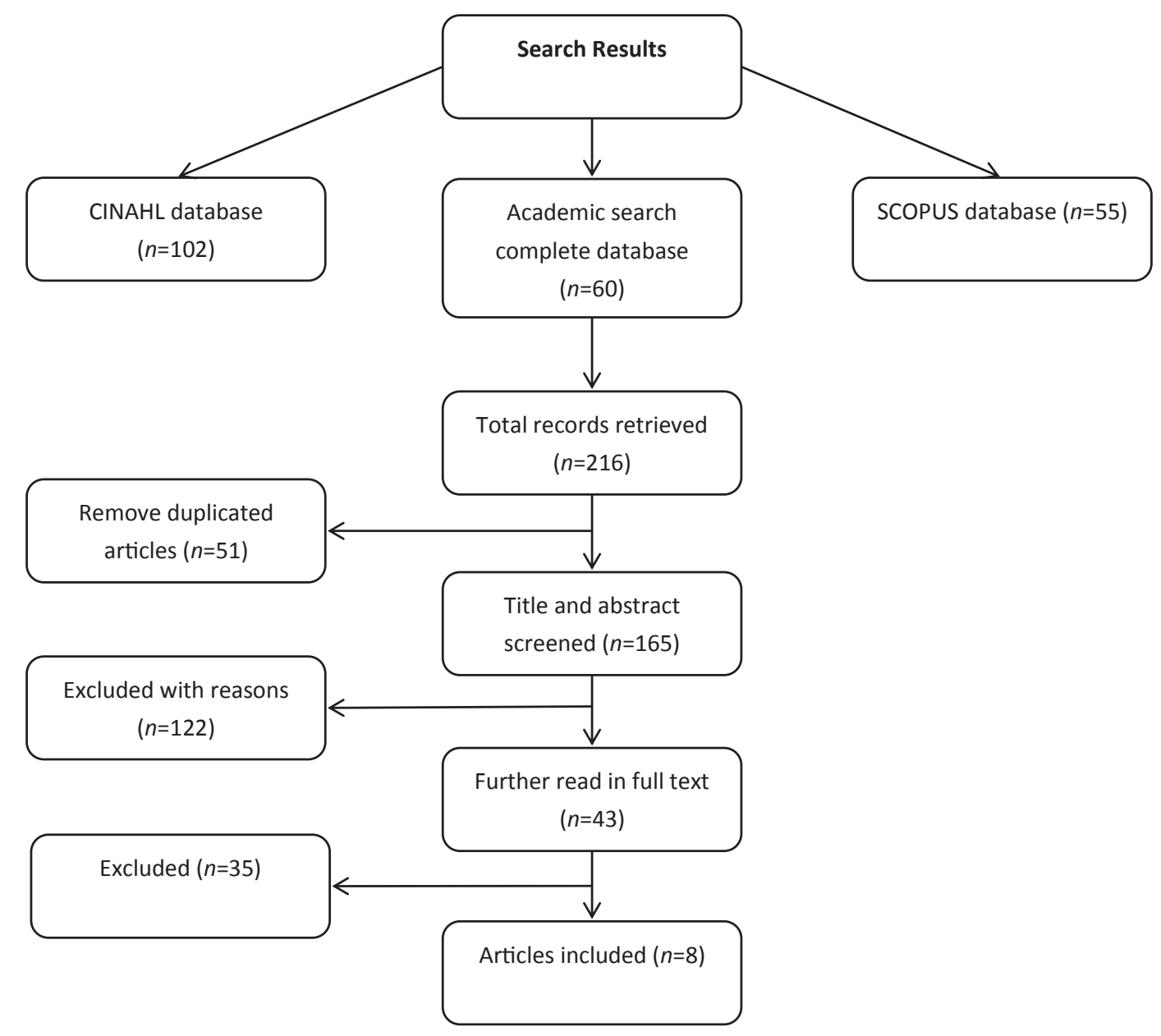

Figure 3. A detailed flow chart of the search process 
ment of medical technology and many changes in the nursing workplace, ${ }^{17}$ the findings of these review articles are too old to reflect the current experiences of overseas nurses. Additionally, their research used a qualitative approach, but their findings were merely extracted from qualitative research. By contrast, by adopting a systematic approach, this literature review further explores this topic from both qualitative and quantitative perspectives, thus providing a more comprehensive understanding of both the negative and positive aspects of overseas nurses' experiences.

\subsection{Research questions}

(1) What challenges do overseas nurses experience during their working careers in the UK?

(2) What support is available to them?

\subsection{Research objectives}

(1) To critically explore the challenges faced by overseas nurses during their working careers in the UK;

(2) to critically explore the support available to overseas nurses during their working careers in the UK; and

(3) to make recommendations on how to minimize the effects of challenges and provide more support to overseas nurses during their working careers in the UK.

\section{Methodology}

This study critically evaluates the relevant literature, including qualitative research, quantitative research, and mixed-method research, by following an all-around search strategy. It also conducts a critical appraisal of the relevant literature. In addition, adopting a systematic approach to the literature review enables the reader to be confident that a thorough search has been carried out and that the results and conclusions are reliable. ${ }^{18}$ Hence, this literature review adopts a systematic approach to explore challenges and available support that overseas nurses are likely to experience during their working careers in the UK.

\subsection{Data collection}

\subsubsection{Databases}

The literature was identified through 3 appropriate electronic online databases: the Cumulative Index to Nursing and Allied Health Literature (CINAHL),
Scopus, and Academic Search Complete databases. Inclusion and exclusion criteria are fundamental for ensuring that the literature is searched in a systematic manner. Simpson et al. ${ }^{19}$ suggested that researchers should develop inclusion and exclusion criteria on the basis of the research questions. Hence, in this review, the inclusion and exclusion criteria were designed as a response to the research questions "What challenges do overseas nurses experience during their working careers in the UK? What support is available to them?" (Table 1). Additionally, the Population Intervention Comparison Outcome (PICO) model was used throughout the search. The PICO format is regarded as a valid strategy for framing a prospect concerning research questions and makes the search more accurate. By using the PICO model, key concepts in the research questions were identified. Furthermore, appropriate search terms to describe the inclusion and exclusion criteria were developed.

Only articles published within the past 16 years were included due to the rapid changes in the innovation of medical technologies and policies. ${ }^{17}$ To ensure an analysis of current experiences of overseas nurses working in the UK, the searches were confined to articles published in recent years. Importantly, there was a rapid increase in the number of the UK overseas nurses from European countries and other countries in the late 1990s and earlier years of this decade. ${ }^{20}$ This trend lasted for a long time, until 2002. In 2002, the number of nursing registrations showed an apparent decline for the first time. Since 2002, there has been a marked reduction in overseas registrants. ${ }^{20}$ The reasons for this reduction are worth researching. To a large extent, this phenomenon may be associated with the challenges of working abroad and the lack of support mechanisms available to overseas nurses in the UK. Hence, the information yield was limited to the years 2002-2017. Though not ideal, limiting the search by date was necessary due to time constraints for the completion of the literature review. A detailed flow chart of the search process is presented herein (Figure 3).

\subsection{Framework for critical appraisal}

The reviewer adopted the Critical Appraisal Skills Programme (CASP) and Critical Appraisal of a Descriptive Study to appraise selected articles. CASP, produced by Oxford University, offers a convenient and effective method to critically appraise diverse study methods. ${ }^{21}$ Eight CASP appraisal tools were suitable to differentiate between the study designs of the articles to be appraised. One of these tools was included in the CASP Qualitative Checklist, which is useful to appraise the 
quality of qualitative research. The reviewer used the CASP Qualitative Checklist to assess the 6 selected qualitative research studies. The quality and rigor of the methodology of these qualitative research studies was examined. Additionally, the Critical Appraisal of a Descriptive Study is a useful measure of the methodological quality of a given descriptive study. The 11 questions in this appraisal were particularly valid due to the detailed explanation of the criteria for every item. In the case of purely quantitative research, the reviewer used this appraisal approach. Importantly, appraisal of mixedmethod research articles was conducted with both the CASP Qualitative Checklist and Critical Appraisal of a Descriptive Study. A summary of the assessment of each of the articles selected using these tools can be found in Appendix 1.

\subsection{Data extraction}

Gathering studies in a generic format can simplify data presentation and make data synthesis easier. In this review, the following pieces of information were extracted from the 8 selected articles: author, research focus, sampling method, location/setting, and type of research methods (Table 2).

\subsection{Ethical considerations}

In accordance with the research guidelines, ethical considerations were undertaken as required. Since this review was based on secondary data and did not recruit individual participants, it involved a very low risk from an ethical standpoint. In addition, among the 8 selected articles, 7 included clear ethics considerations and obtained ethical approval from specific agencies or committees.

\section{Results}

The findings of the articles were grouped into themes (Table 3). By summarizing the results of these studies, an overview of their similarities and inconsistencies is clearly presented. This table shows that the role of gender in migrant nurses working in the UK was only researched by 1 article, written by Winkelmann-Gleed and Seeley. ${ }^{28}$ Thus, due its limited assessment, this theme was eliminated from further discussion in this review. Furthermore, most of the articles discussed cultural differences, communication issues, and unequal treatment in the British health-care system. Half of the articles included a detailed discussion on de-skilling. In

\begin{tabular}{|c|c|c|c|c|c|c|}
\hline Author & Research focus & Location & $\begin{array}{c}\text { Numbers of } \\
\text { Samples }\end{array}$ & Quantitative & Qualitative & Study design \\
\hline $\begin{array}{l}\text { Alexis \& Vydelingum, } \\
200422\end{array}$ & $\begin{array}{l}\text { Experiences of black and } \\
\text { minority ethnic overseas } \\
\text { nurses }\end{array}$ & $\mathrm{NHS}$ & 12 & & $\sqrt{ }$ & Interviews \\
\hline Alexis, 201323 & $\begin{array}{l}\text { Experiences of } \\
\text { internationally educated } \\
\text { nurses }\end{array}$ & England & 35 & & $\sqrt{ }$ & $\begin{array}{l}\text { Interviews, } \\
\text { focus group }\end{array}$ \\
\hline Henry, 200724 & $\begin{array}{l}\text { Overseas-trained nurses' } \\
\text { experiences of career } \\
\text { progression }\end{array}$ & $\mathrm{NHS}$ & 20 & & $\sqrt{ }$ & $\begin{array}{l}\text { Interviews, } \\
\text { field work }\end{array}$ \\
\hline $\begin{array}{l}\text { Matiti and Taylor, } \\
200515\end{array}$ & $\begin{array}{l}\text { Cultural backgrounds of } \\
\text { internationally recruited } \\
\text { nurses affect their } \\
\text { adjustment process; } \\
\text { identifying support } \\
\text { mechanisms }\end{array}$ & UK & 12 & & $\sqrt{ }$ & Interviews \\
\hline Taylor, 200525 & $\begin{array}{l}\text { Experiences of nurses who } \\
\text { have trained overseas }\end{array}$ & UK & 11 & & $\sqrt{ }$ & Focus group \\
\hline $\begin{array}{l}\text { Alexis and Shillingford, } \\
201126\end{array}$ & $\begin{array}{l}\text { The perceptions and } \\
\text { work experiences of } \\
\text { internationally recruited } \\
\text { neonatal nurses }\end{array}$ & $\mathrm{NHS}$ & 13 & & $\sqrt{ }$ & Interviews \\
\hline Alexis, 201527 & $\begin{array}{l}\text { Internationally recruited } \\
\text { nurses perceive } \\
\text { discrimination and support } \\
\text { in the workplace }\end{array}$ & $\mathrm{NHS}$ & 188 & $\sqrt{ }$ & & Questionnaire \\
\hline $\begin{array}{l}\text { Winkelmann-Gleed \& } \\
\text { Seeley, } 200528\end{array}$ & $\begin{array}{l}\text { Experiences of recently } \\
\text { internationally qualified } \\
\text { migrant nurses to Britain }\end{array}$ & London & 22 & $\sqrt{ }$ & $\sqrt{ }$ & $\begin{array}{l}\text { Survey } \\
\text { interviews }\end{array}$ \\
\hline
\end{tabular}

Table 2. Data extracted from the 8 selected articles 


\begin{tabular}{|c|c|c|c|c|c|c|c|}
\hline \multirow[b]{2}{*}{ Authors } & \multicolumn{5}{|c|}{ Challenges } & \multicolumn{2}{|c|}{ Support } \\
\hline & $\begin{array}{c}\text { Cultural } \\
\text { differences }\end{array}$ & $\begin{array}{c}\text { Communication } \\
\text { issues }\end{array}$ & Gender & $\begin{array}{l}\text { Unequal } \\
\text { treatment }\end{array}$ & De-skilling & Formal & Informal \\
\hline $\begin{array}{l}\text { Alexis \& Vydelingum, } \\
2005^{22}\end{array}$ & & & & $\sqrt{ }$ & & & $\sqrt{ }$ \\
\hline Alexis, $2013^{23}$ & $\sqrt{ }$ & $\sqrt{ }$ & & $\sqrt{ }$ & & & $\sqrt{ }$ \\
\hline Henry, $2007^{24}$ & $\sqrt{ }$ & $\sqrt{ }$ & & $\sqrt{ }$ & & $\sqrt{ }$ & $\sqrt{ }$ \\
\hline Matiti and Taylor, $2005^{15}$ & $\sqrt{ }$ & $\sqrt{ }$ & & & $\sqrt{ }$ & $\sqrt{ }$ & $\sqrt{ }$ \\
\hline Taylor, $2005^{25}$ & $\sqrt{ }$ & $\sqrt{ }$ & & $\sqrt{ }$ & $\sqrt{ }$ & $\sqrt{ }$ & $\sqrt{ }$ \\
\hline $\begin{array}{l}\text { Alexis and Shillingford, } \\
2011^{26}\end{array}$ & $\sqrt{ }$ & $\sqrt{ }$ & & $\sqrt{ }$ & $\sqrt{ }$ & & $\sqrt{ }$ \\
\hline Alexis, $2015^{27}$ & & & & $\sqrt{ }$ & & & $\sqrt{ }$ \\
\hline $\begin{array}{l}\text { Winkelmann-Gleed \& } \\
\text { Seeley, } 2005^{28}\end{array}$ & $\sqrt{ }$ & & $\sqrt{ }$ & $\sqrt{ }$ & $\sqrt{ }$ & & $\sqrt{ }$ \\
\hline
\end{tabular}

Table 3. Summarized findings of the 8 selected articles

addition, sources of formal and informal support, which can help to relieve the effects of these negative factors, were clearly identified in each article. Hence, except for the role of gender, the other 6 emerging themes were further analyzed by contrasting and integrating the different viewpoints of these authors.

\section{Discussion}

\subsection{Theme 1 - cultural differences}

One pressing challenge encountered by overseas nurses was cultural differences. Being from a different part of the world, overseas nurses had experienced a different culture. Alexis, ${ }^{23}$ Matiti and Taylor, ${ }^{15}$ Alexis and Shillingdord, ${ }^{26}$ Taylor, ${ }^{25}$ and Winkelmann-Gleed and Seeley ${ }^{28}$ all focused on this theme, showing the negative effects of cultural differences on the experiences of overseas nurses. Alexis ${ }^{23}$ highlighted the effects of the culture shock experienced by overseas nurses. He found that these overseas nurses had to learn a new approach to patient care due to differences between the British health-care systems and their former health-care system. For example, overseas nurses may be familiar with the medical equipment used in their country of origin but may not be familiar with the equipment used in the UK. Additionally, overseas nurses from North America were accustomed to standard approbation but had to change this usual practice into documentation because there is no standard approbation or a nationally agreedon approbation in the UK.

Similarly, both Matiti and Taylor ${ }^{15}$ and Alexis and Shillingdord ${ }^{26}$ also acknowledged that internationally recruited nurses often experience cultural shock while adapting to their new life and new workplace. Matiti and
Taylor ${ }^{15}$ noted that nursing practices were new to them, ranging from basic practices, such as cleaning patients after defecation and bed baths, to complex practices, such as using Dynamap and percutaneous endoscopic gastrostomy feeding. Additionally, the degree of paperwork required by the UK medical system is often reported as being overwhelming to overseas-trained nurses, and they often must spend much more time on paperwork than on providing actual patient care. ${ }^{15}$ Moreover, the National Service Framework for Children advocates that family-centered care should be of primary importance in the delivery of care and all nurses must align their care according to this framework. ${ }^{29}$ However, the findings of Alexis and Shillingdord's study ${ }^{26}$ revealed that internationally recruited neonatal nurses were not familiar with the family-centered care system emphasized in the UK. The nurses often found it challenging to adjust to a system in which health education was provided to the family during the care of neonates. Alexis and Shillingdord ${ }^{26}$ highlighted how this unfamiliarity can directly influence the quality of patient care.

In addition, Taylor ${ }^{25}$ noted that differences in nursing roles created many barriers to overseas nurses' work, especially when caring for elderly patients. For instance, in Nigeria, the role of the family includes caring for the elderly in the community settings. By contrast, in the UK, community nurses play a more important role in caring for the elderly who are waiting for a residential home placement. Surprisingly to these nurses, the family was included in patient care in the UK. Apart from new ways to perform patient care and different nursing roles, the different levels of autonomy in decision-making also required these overseas nurses to adapt to the ward culture of the UK. ${ }^{25}$ Thus, due to cultural differences, it is quite challenging for overseas nurses to adapt to the values and norms that underpin the British health-care system. ${ }^{28}$ 


\subsection{Theme 2 - communication issues}

Differences in accent and pronunciation restrict overseas nurses' understanding and expression. ${ }^{30}$ Likewise, to these nurses, understanding the sociocultural aspects of communication is very challenging. Previous findings ${ }^{15,23-25,28}$ acknowledged that the effects of communication issues in nursing practices were evident for overseas nurses. Alexis ${ }^{23}$ reported that internationally educated nurses felt isolated when they were unable to communicate fluently with patients and host nurses. Taylor ${ }^{25}$ strongly supported this viewpoint. He also found that these nurses not only experienced difficulties in communication but were also isolated by UK nurses. Full integration into the ward is not possible for these overseas nurses when their capacity to communicate is restricted. In addition, Matiti and Taylor ${ }^{15}$ reported that internationally recruited nurses could often not even understand other foreign nurses' accents. They also found that some internationally recruited nurses could not answer the phone due to comprehension issues. The absence of nonverbal cues makes telephone communication extremely stressful given their fear of miscommunication and subsequent harm to patients. ${ }^{15}$ Importantly, communication issues negatively contribute to career development to a large extent. ${ }^{24}$ Some overseas nurses had difficulties in applying current buzzwords and professional language to express themselves in applications or during interviews. Henry ${ }^{24}$ also found that some African nurses' career stagnation was due to their poor communication skills compared to their UK-trained colleagues. The importance for adhering to professional discourses and improving communication skills in achieving successful career development was also highlighted in Henry's ${ }^{24}$ research.

However, Winkelmann-Gleed and Seeley ${ }^{28}$ believed that language issues can function as a double-edged sword. On the one hand, since many overseas nurses speak English as a second language, rather than their mother tongue, cross-cultural communication issues can arise. On the other hand, overseas nurses recognize the value of their language skills when communicating with minority ethnic patients, especially in multicultural parts of the UK, such as London.

\subsection{Theme 3 - unequal treatment}

Another pertinent theme regarding the challenges experienced by overseas nurses focused on equity. In the UK, unequal treatment in the workplace was commonly reported by overseas nurses. ${ }^{22-25,27,28}$ Alexis $^{23}$ noted that many internationally educated nurses encountered marginalization in the form of separation. Moreover, overseas nurses described that they felt unappreciated although they worked extra shifts. ${ }^{22}$ That is, they felt that their colleagues and managers did not appreciate the hard work that they performed. Alexis and Vydelingum ${ }^{22}$ also found that often, overseas black and minority ethnic nurses were given the most difficult tasks. Sometimes, a few senior staff members make more demands of foreign nurses than UK-trained nurses. Despite these poor conditions, foreign-trained nurses had little choice but to accept this treatment, as they often have had few other opportunities. They were also unwilling to stand up to authority. Winkelmann-Gleed and Seeley ${ }^{28}$ also found that overseas nurses often felt helpless when they experienced unfair work assignments and requirements.

In addition, a number of international nurses claimed that they had no opportunities for career development. ${ }^{28}$ Henry $^{24}$ as well as Alexis and Vydelingum ${ }^{22}$ confirmed this finding. Henry ${ }^{24}$ reported that the process of promotion often lacked transparency. He also wrote that these nurses could not gain management experience and had no access to appropriate training compared with UKtrained nurses. Importantly, Alexis and Vydelingum ${ }^{22}$ also stated that their ethnic identity led to the refusal of training to some extent. They wrote that overseas nurses did not have access to a training course to develop themselves, even though additional training is a requirement for skill development and career progression.

Additionally, Alexis and Vydelingum ${ }^{22}$ attributed this unfairness to the British health-care system's hierarchical nature. Other studies identified discrimination as a major reason for this unequal treatment. ${ }^{25,27}$ Alexis $^{27}$ stated that internationally recruited nurses were discriminated against in the clinical environment $(P<0.000)$. It was found that their experiences were largely different from those of UK-trained nurses. The hospital staff, patients, and their relatives were often aggressive toward them through harmful or offensive actions. Moreover, Taylor ${ }^{25}$ illustrated that racial discrimination was apparent in the NHS. He found that the humor used in the workplace referred to overseas nursing practices as being inferior. In response to this, many overseas nurses also used humor to suggest that the UK technology was inferior. Both groups of nurses used this method to highlight their differences, which was harmful for the creation of a multicultural workplace.

\subsection{Theme 4 - de-skilling}

Some of the selected studies identified the resentment and frustration felt by overseas nurses when their skills and knowledge were questioned by their UK counterparts. ${ }^{15,22,25,26,28}$ Winkelmann-Gleed and Seeley ${ }^{28}$ found that migrant nurses were always regarded as student 
nurses, although they come to the UK with years of professional experience. Worse, the feelings of being treated as a novice in the workplace had a negative influence on their confidence and self-esteem. ${ }^{26}$ Alexis and Vydelingum's ${ }^{22}$ study also confirmed this impact on internationally recruited nurses. They ${ }^{22}$ noted that it is important to recognize the clinical competence and value the knowledge that overseas nurses had already acquired. They also reported that overseas nurses' experience can benefit the British nursing system, rather than their services being viewed only as a crisis intervention to rectify the shortage of nurses.

Additionally, Matiti and Taylor ${ }^{15}$ noted that nurses hoped that the skills they were accustomed to in their home country could still be used in the UK, but they could not use these skills due to policy restrictions. They found that these nurses felt confused about which nursing practices they were allowed to use in different wards. The "nursing" that they valued and thought was nursing was not recognized. This was a challenge to what they perceived as "nursing". ${ }^{15}$ There was thus a mismatch between the organizational values and individual nurses' values. Moreover, Taylor ${ }^{25}$ wrote that professional recognition of their qualifications did not automatically mean that their clinical competence and knowledge were recognized. This was not only indicated in the lowest grade for qualified nurses but was also reflected in their tasks. For example, they were often asked to assist patients with personal care rather than be involved in catheterization or venipuncture, which are often viewed as basic skills in their country. Taylor $^{25}$ noted that this assumed ignorance denied their skills and experience the recognition they deserved and served to reinforce their junior status.

\subsection{Theme 5 - formal support}

Some of the challenges experienced by overseas nurses are largely associated with technical issues and culture. These technical problems, such as challenges in interviewing or producing a curriculum vitae, can be solved by formal training and mentoring. ${ }^{24}$ Before these nurses work in the UK, it is necessary and helpful to provide support in the preparation and adaptation processes. ${ }^{15,24,25}$ Taylor $^{25}$ stated that an adaptation program can be one of the best methods of support to help overseas nurses become familiar with the British health-care system. In addition, Matiti and Taylor ${ }^{15}$ noted that internationally recruited nurses could go to the British Embassy to acquire important documents, such as the NMC information sheet and a working permit. Apart from this, induction programs from the hospitals or other agencies often play a vital role for these nurses. ${ }^{15}$ However, most orientation programs did not focus on the specific cultural needs of internationally recruited nurses. ${ }^{15}$ They instead emphasized the necessity of culturally oriented information provided by various sources in minimizing "culture shock". Moreover, Matiti and Taylor $^{15}$ and Henry ${ }^{24}$ argued that accommodation issues also need to be addressed immediately after professional training programs, as well as providing sufficient informational support. Appropriate accommodation offered by employers can make their adaptation to the new environment easier. In addition, Taylor ${ }^{25}$ wrote that some special arrangements, such as matching their shifts to their partner's shift patterns on another ward and coordinating off-duty time to ensure access to public transport, should be included in the support package.

Although some logistical support, such as help with accommodations and airport greetings, has been provided, several studies have suggested that foreigntrained nurses have limited access to equal, adequate, and transparent formal support. ${ }^{15,24}$ Specifically, the findings of Henry ${ }^{24}$ noted that these nurses had to repeatedly request formal support and when they did receive it, it was often interior to the support given to UK-trained nurses.

\subsection{Theme 6 - informal support}

Alexis ${ }^{27}$ stated that internationally recruited nurses could receive support $(P<0.001)$ in the clinical environment. Although this research did not mention which type of support, it demonstrated that the support received by African-trained nurses was minimal compared to that of nurses trained in other countries.

All of the reviewed articles highlighted the importance of informal networks and social support systems for adapting to new environments. Taylor, Henry, Alexis, Winkelmann-Gleed and Seeley, and Alexis and Shillingdord all admitted that the formal systems of support were not major sources of help to foreigntrained nurses and that the far more effective support system was composed of their colleagues from the UK and overseas. ${ }^{23-26,28}$ Despite this, there were some differences between the supportive populations. For example, Winkelmann-Gleed and Seeley ${ }^{28}$ illustrated that most overseas nurses were more willing to ask for help from more supportive senior nurses, while Alexis ${ }^{23}$ found that overseas nurses preferred to seek support from nurses of the same color than from their senior nurses or managers. Importantly, Alexis and Shillingdord $^{26}$ stated that, although their UK-trained colleagues could provide mixed support, the support was unable to fully guide foreign-trained nurses or provide them with valid information for caring. Importantly, the experiences 
of other overseas nurses were more effective and valuable in minimizing culture shock because of their similar cultural background and communication issues. In addition, the findings of Taylor ${ }^{25}$ indicated that these overseas nurses could benefit from pastoral support from their UK colleagues. Indeed, their local coworkers could be of great help by doing such things as taking them to areas of local or scenic interest on their day off or driving them home.

However, in contrast to these 5 studies, Alexis and Vydelingum $^{22}$ deemed that this type of support mostly came from their shared affinity with other black and minority ethnic nurses. They found that the absence of support from UK-trained white nurses caused a large amount of difficulties and produced an unpleasant workplace environment. In addition, black and ethnic minority nurses were more willing to care for each other without the participation of white nurses. This type of collaborative environment, even if it does not cross racial or ethnic lines, may still lead to stress reduction and increased clinical confidence.

\section{Conclusions}

As foreign-trained nurses make up an increasing proportion of the UK workforce, developing a full picture of their negative and positive experiences is becoming increasingly critical. ${ }^{20}$ Importantly, although these overseas nurses have made great contributions to patient care within the British health-care system, their experiences during their working careers in the UK are not always positive. ${ }^{23}$ Current health policies place a strong emphasis on diversity and inclusion and require the creation of a more supportive workplace for overseas nurses. ${ }^{20}$ This study provided insight into the challenges experienced by overseas nurses during their working careers in the UK. This study also provided an overview of the formal and informal support systems available to overseas nurses in the UK.

\section{Limitations}

Researcher bias occurs when a researcher unconsciously affects the results or data interpretation due to subjective influences, and it is often difficult for researchers to be entirely objective and to ensure that they are not being influenced by personal emotions, desires, or biases. In this review, researcher bias may have affected the topic choice and selection of the reviewed articles. For instance, based on the limited financial support, relevant studies were likely to be missed because they were unavailable from the Online Hospital Library. Researcher bias also likely influenced the eventual findings to some extent due to the researcher's subjective desires.

Another limitation of this review is the possibility that inappropriate data were included in the reviewed studies. The data in some of these reviewed articles were collected $>10$ years ago. With the rapid development of medical techniques in the UK, ${ }^{17}$ these articles may therefore not reflect the current situation. In addition, the data in this review were collected and summarized from studies from different regions in the UK. Due to some regional policy independences and different economic levels, the challenges and available supports in this review may not be generalizable for all regions. Furthermore, due to different health-care systems and cultures in their countries of origin, the experiences of overseas nurses are extremely diverse. Because the participants in 1 reviewed article ${ }^{24}$ were from Ghana, some findings in this study may merely reflect the experiences of Ghanaian nurses and may not be generalizable to all foreign-trained nurses.

Additionally, the population of overseas nurses working in the UK is quite large, but the total participants in these reviewed articles accounted for much less than $1 \%$ of the total population. Furthermore, because several of the included studies mostly included volunteer participants, it is possible that a volunteer bias skewed the perspectives of those included. Indeed, the views of these volunteers may not be reflective of the entire population of nurses in the UK. However, there is a sharp distinction between overseas nurses who are willing to participate in research and those who do not wish to do so. ${ }^{31}$ Those who had bad experiences tend to be more interested in the topic and are more likely to volunteer ${ }^{31}$ because they would like to express their dissatisfaction and help to combat the situation. Hence, the findings may not be reflective of the experiences of all overseas nurses in the UK.

\section{Recommendations}

Policymakers play a key role in ensuring that evidencebased policies are revised for the benefit of overseas nurses, as well as for sustainability and effectiveness in the workplace. ${ }^{26}$ With the transformation of the current national and global nursing labor market, they must evaluate the benefits and drawbacks of recruiting overseas nurses and conduct a comprehensive costbenefit analysis. Moreover, regular revision of policies and strategies is required to promote both long-term and immediate interests and to balance benefits to the health-care system with benefits to individual foreigntrained nurses. In addition, more attention should be required in the de-skilling of overseas nurses at the level of national policy. The international recruitment of health 
workers is viewed as a 2-way exchange of expertise. ${ }^{32}$ Overseas nurses have various unique skills and talents, which they can provide to the health-care services; however, they cannot make full use of their experiences and skills in the UK. Hence, it is necessary for national and local policymakers to formulate innovative strategies or set up special standards to reduce the de-skilling of overseas nurses. Such changes will eventually allow them to freely provide high-quality care.

From a managerial perspective, employers must offer equal opportunities and treatment to every nurse in tandem with existing employment laws, policies, and regulations in the host countries. ${ }^{33}$ Furthermore, employers cannot group all overseas nurses together as a seemingly homogeneous group, which is critical in creating a multicultural and friendly work environment. More importantly, employers could strengthen the enforcement of policies and regulations of various agencies.

In addition, organizational interventions are necessary to improve the long-term retention of foreign-trained

\section{References}

1. International Organization for Migration. World Migration Report; 2013. http://publications.iom.int/system/ files/pdf/wmr2013_en.pdf. Accessed August 23, 2010.

2. World Health Organization. Migration of health workers. http://www.who.int/hrh/migration/14075_MigrationofHealth_Workers.pdf. Accessed March 20, 2010.

3. Buchan J. International recruitment of nurses: policy and practice in the United Kingdom. Health Serv Res. 2007;42:1321-1335.

4. Marsh B, Loudon A. Half of the NHS's new nursing recruits are from overseas. http://www.dailymail. co.uk/news/article-61795/Half-NHSs-new-nursingrecruits-overseas.html. Accessed March 20, 2010.

5. Buchan J, Seccombe I. The UK nursing labour market review 2012. UK Nursing Labour Market; 2012.

6. Lintern S. NHS to face chronic nurse shortage by 2016. http://www.nursingtimes.net/roles/nurse-managers/ nhs-to-face-chronic-nurse-shortage-by-2016/ 5059871.fullarticle. Accessed June 18, 2013.

7. Marangozov R, Williams M, Buchan J. The Labour Market for Nurses in the UK and its Relationship to the Demand for, and Supply of, International Nurses in the NHS. United Kingdom: Migration Advisory Committee; 2016.

8. Sochan A, Singh MD. Acculturation and socialization: voices of internationally educated nurses in Ontario. Int Nurs Rev. 2007;54:130-136.

9. Nichols J, Campbell J. Experiences of overseas nurses recruited to the NHS: Julia Nichols and nurses. ${ }^{34}$ Overseas nurses should receive compulsory training and assessment before managers ask them to perform nursing duties. Even well-structured orientation and mentoring programs do not fully satisfy their needs. Multifaceted instruction on equipment and technology as well as ongoing cultural training ought to be involved. Investing resources in orientation support for qualified foreign-trained nurses may give them greater access to the benefits of the system while allowing them to contribute more to British health care. ${ }^{16}$ Furthermore, to ensure that overseas nurses are proportionately represented at senior levels, human resource departments should conduct longtime monitoring of career development. Meanwhile, similar monitoring should be conducted to ensure that each overseas nurse has the opportunity to obtain the necessary training to successfully apply for senior positions.

\section{Conflicts of interest}

All contributing authors declare no conflicts of interest.
Jackie Campbell discuss findings from an integrative review of the opinions of internationally recruited nurses who have worked in the UK. Nurs Manag. 2010;17:30-35.

10. Buchan J, Dovlo D. International Recruitment of Health Workers to the UK: A Report for DFID. London: DFID Health Systems Resource Centre; 2004.

11. Kovner CT, Brewer CS, Fatehi F, Jun J. What does nurse turnover rate mean and what is the rate? Policy Polit Nurs Pract. 2014;15:64-71.

12. Stuart P. Overseas nurses' experience as support workers in the UK. Nurs Resident Care. 2012;14:660-663.

13. Okougha M, Tilki M. Experience of overseas nurses: the potential for misunderstanding. $\mathrm{Br} J$ Nurs. 2010;19:102-106.

14. Withers J, Snowball J. Adapting to a new culture: a study of the expectations and experiences of Filipino nurses in the Oxford Radcliffe Hospitals NHS Trust. Nurs Times Res. 2003;7:278-290.

15. Matiti MR, Taylor D. The cultural lived experience of internationally recruited nurses: a phenomenological study. Divers Equal Health Care. 2005;2:7-16.

16. Nichols J, Campbell J. The experiences of internationally recruited nurses in the UK (1995-2007): an integrative review. J Clin Nurs. 2010;19:2814-2823.

17. Bergsland J, Elle OJ, Fosse E. Barriers to medical device innovation. Med Devices (Auckl). 2014;7: 205-209. 
18. Aveyard H. Doing A Literature Review in Health and Social Care. Maidenhead, Berkshire, England: McGraw-Hill/Open University Press; 2010.

19. Simpson F, Sweetman EA, Doig GS. A systematic review of techniques and interventions for improving adherence to inclusion and exclusion criteria during enrolment into randomised controlled trials. Trials. 2010;11:17.

20. Royal College of Nursing. International Recruitment 2015. https://www2.rcn.org.uk/_data/assets/pdf_ file/0007/629530/International-Recruitment-2015. pdf. Accessed June 15, 2015.

21. Taylor J. Doing a literature review in health and social care: a practical guide, 2nd edition [book review]. Health Inform. 2010;19:24.

22. Alexis $O$, Vydelingum $V$. The experiences of overseas black and minority ethnic nurses in the NHS in an English hospital: a phenomenological study. J Res Nurs. 2005;10:459-472.

23. Alexis O. Internationally educated nurses' experiences in a hospital in England: an exploratory study. Scand J Caring Sci. 2013;27:962-968.

24. Henry L. Institutionalized disadvantage: older Ghanaian nurses' and midwives' reflections on career progression and stagnation in the NHS. J Clin Nurs. 2007; 16:2196-2203.

25. Taylor B. The experiences of overseas nurses working in the NHS: results of a qualitative study. Divers Health Social Care. 2005;2:17-27.

26. Alexis O, Shillingford A. Exploring the perceptions and work experiences of internationally recruited neonatal nurses: a qualitative study. J Clin Nurs. 2012;21:1435-1442.

27. Alexis O. Internationally recruited nurses' experiences in England: a survey approach. Nurs Outlook. 2015;63:238-244.

28. Winkelmann-Gleed A, Seeley J. Strangers in a British world? Integration of international nurses. Br J Nurs. 2005;14:954-961.

29. Department of Health. National Service Framework for Children, Young People and Maternity Services. https://www.gov.uk/government/uploads/system/ uploads/attachment_data/file/199952/National_ Service_Framework_for_Children_Young_People_ and_Maternity_Services_-_Core_Standards.pdf. Accessed October 4, 2004.

30. Singleton K, Krause EM. Understanding cultural and linguistic barriers to health literacy. Kentucky Nurse. 2010;58:4-9.

31. Heiman GW. Research Methods in Psychology. 3rd ed. Boston \& New York: Houghton Mifflin Co; 2002.

32. Gerrish K, Griffith V. Integration of overseas Registered Nurses: evaluation of an adaptation programme. J Adv Nurs. 2004;45:579-587.

33. Hagey R, Choudhry U, Guruge S, Turrittin J, Collins $E$, Lee R. Immigrant nurses' experience of racism. J Nurs Scholarsh. 2001;33:389-394.

34. Jeans H, Hadley F, Green J, et al. Navigating to Become a Nurse in Canada: Assessment of International Nurse Applicants. https://www.cna-aiic. $\mathrm{ca} / \sim / \mathrm{media} / \mathrm{cna} /$ page-content/pdf-fr/ien_technical_ report_e.pdf?la=en. Accessed August 23, 2010.

How to cite this article: Lin YQ, Ding Y, Li JY. A literature review of research exploring the experiences of overseas nurses in the United Kingdom (2002 -2017). Frontiers Nurs. 2018; 1: 17-30. https://doi.org/10.1515/ fon-2018-0003 


\section{Appendix 1: Appraisal tools}

(1) Critical Appraisal Skills Programme (CASP) Qualitative Checklist

Full checklist link: http://media.wix.com/ugd/dded87_29c5b002d99342f788c6ac670e49f274.pdf

\begin{tabular}{|c|c|c|c|c|c|c|c|}
\hline \multirow{2}{*}{$\begin{array}{l}\text { CASP Qualitative } \\
\text { Checklist } \\
\text { Author Questions 1-9 }\end{array}$} & \multicolumn{6}{|c|}{ Qualitative research } & \multirow{2}{*}{$\begin{array}{c}\begin{array}{c}\text { Mixed- } \\
\text { method } \\
\text { research }\end{array} \\
\text { Winkelmann- } \\
\text { Gleed and } \\
\text { Seeley }(2005) \\
\end{array}$} \\
\hline & $\begin{array}{c}\text { Alexis and } \\
\text { Vydelingum } \\
\text { (2005) }\end{array}$ & $\begin{array}{l}\text { Alexis } \\
(2014)\end{array}$ & $\begin{array}{l}\text { Henry } \\
(2007)\end{array}$ & $\begin{array}{l}\text { Matiti and } \\
\text { Taylor } \\
\text { (2005) }\end{array}$ & $\begin{array}{l}\text { Taylor } \\
(2005)\end{array}$ & $\begin{array}{l}\text { Alexis and } \\
\text { Shillingford } \\
(2011)\end{array}$ & \\
\hline $\begin{array}{l}\text { 1. Was there a clear statement of } \\
\text { the aims of the research? }\end{array}$ & Yes & Yes & Yes & Yes & Yes & Yes & Yes \\
\hline $\begin{array}{l}\text { 2. Is a qualitative methodology } \\
\text { appropriate? }\end{array}$ & Yes & Yes & Yes & Yes & Yes & Yes & Yes \\
\hline $\begin{array}{l}\text { 3. Was the research design } \\
\text { appropriate to address the } \\
\text { aims of the research? }\end{array}$ & Yes & Yes & Yes & Yes & Yes & Yes & Yes \\
\hline $\begin{array}{l}\text { 4. Was the recruitment strategy } \\
\text { appropriate to the aims of the } \\
\text { research? }\end{array}$ & Yes & Yes & Yes & Yes & Yes & No & Yes \\
\hline $\begin{array}{l}\text { 5. Was the data collected in } \\
\text { a way that addressed the } \\
\text { research issue? }\end{array}$ & Yes & Yes & Yes & Yes & Yes & Yes & Yes \\
\hline $\begin{array}{l}\text { 6. Has the relationship between } \\
\text { researcher and participants } \\
\text { been adequately considered? }\end{array}$ & No & No & No & No & Yes & Yes & Yes \\
\hline $\begin{array}{l}\text { 7. Have ethical issues been } \\
\text { taken into consideration? }\end{array}$ & Yes & Yes & No & Yes & Yes & Yes & Yes \\
\hline $\begin{array}{l}\text { 8. Was the data analysis } \\
\text { sufficiently rigorous? }\end{array}$ & Yes & Yes & Yes & Yes & Yes & Yes & No \\
\hline $\begin{array}{l}\text { 9. Is there a clear statement of } \\
\text { findings? }\end{array}$ & Yes & Yes & Yes & Yes & Yes & Yes & Yes \\
\hline
\end{tabular}

\begin{tabular}{|c|c|}
\hline $\begin{array}{l}\text { CASP Qualitative } \\
\text { Checklist }\end{array}$ & Question 10: How valuable is the research? \\
\hline Alexis and Vydelingum (2005) & $\begin{array}{l}\text { This research gave a clear statement of its limitations and discussed its contribution. However, this research } \\
\text { did not discuss the need for dissemination of findings and give any recommendations. }\end{array}$ \\
\hline Matiti and Taylor (2005) & $\begin{array}{l}\text { The necessity of further research to be conducted was highlighted in this research and it identified new areas } \\
\text { that needed exploration. In addition, it listed the most comprehensive recommendations for research by using } \\
4 \text { steps Detailed steps about how to achieve a multicultural nursing work environment were presented at the } \\
\text { end of their research. }\end{array}$ \\
\hline Henry (2007) & $\begin{array}{l}\text { Although this research did not discuss the need for dissemination of findings and give any recommendations, it } \\
\text { made a valuable contribution on overseas nurses' career development. }\end{array}$ \\
\hline Alexis (2014) & $\begin{array}{l}\text { The contribution of this research was to give a more comprehensive understanding of international nurses' } \\
\text { experiences by using a combination of interview and focus group for the first time. Moreover, there was a } \\
\text { critical discussion of the size of sampling and the limitations in dissemination. }\end{array}$ \\
\hline Taylor (2005) & $\begin{array}{l}\text { This research provided valuable resources for improving the work environment of overseas nurses. In } \\
\text { addition, the necessity of further research to be conducted was highlighted, and several recommendations for } \\
\text { employers and researchers were listed clearly. }\end{array}$ \\
\hline Alexis and Shillingford (2011) & $\begin{array}{l}\text { This research gave a clear statement of its limitations and discussed its contribution. Additionally, it gave many } \\
\text { useful advices for policy makers about clinical practice. }\end{array}$ \\
\hline $\begin{array}{l}\text { Winkelmann-Gleed and Seeley } \\
(2005)\end{array}$ & $\begin{array}{l}\text { This study clearly used a complementary strategy, combining a quantitative survey and a qualitative } \\
\text { interview, which provided several findings that were more reliable for further research about overseas nurses' } \\
\text { experiences. However, this study did not give a specific direction on whether its findings can be generalized to } \\
\text { a wider population. }\end{array}$ \\
\hline
\end{tabular}


(2) Quantitative research

Here, 11 questions help you make sense of descriptive/cross-sectional studies. How to use this appraisal tool:

Full checklist link: https://reache.files.wordpress.com/2010/03/cross-sectional-appraisal-tool.pdf

1. Did the study address a clearly focused issue?

Yes

This research aimed to have a good understanding of international nurses' work-related identities and addressed a clearly focused issue.

2. Did the authors use an appropriate method to answer their question?

This research applied mixed methods, combining qualitative and quantitative research, in their research. These methods can provide complementary information that makes up for the shortcomings of using only one method.

3. Were the subjects recruited in an acceptable way?

The participants in the survey were selected through contacts and self-selection.

4. Were the measures accurately measured to reduce bias?

Although the convenience sampling method used in the survey is fast and cost-effective, it can cause selection bias.

5. Were the data collected in a way that addressed the research issue?

This research applied the convenience sampling method and had a clear explanation of the sampling procedure.

6. Did the study have enough participants to minimize the play of chance?

There was a relatively large sample size in this design.

7. How are the results presented, and what is the main result?

$P$-value $<0.05$ was identified as a standard to assess the significance of the difference in the statistics. During the process of thematic analysis, sufficient data were presented in the form of tables to support the findings.

8. Was the data analysis sufficiently rigorous?

This research did not have a clear statement of the data analysis for survey.

9. Is there a clear statement of findings?

The survey included several themes to explain international nurses' experiences in the findings.

10. Can the results be applied to the local population?

This study did not give a specific perspective regarding whether its findings can be generalized to a wider population.

11. How valuable is the research?

The contribution of this research was to provide quantitative evidence about the negative and positive experiences of international nurses. More importantly, through a valuable exploration, this research provided a guide for newcomers to adjust to a new environment.

(3) Mixed-method research

1. Did the study address a clearly focused issue?

The research clearly identified the research populations (internationally registered nurses) and addressed 3 specific research questions, covering support, discrimination, and adaptation to a new workplace.

2. Did the authors use an appropriate method to answer their question?

Alexis (2015) intended to explore the current phenomenon, rather than the causal relationships between the variables. However, experimental design is not suitable for this research. As a result, the author conducted a quantitative descriptive study.

3. Were the subjects recruited in an acceptable way?

This research applied probability sampling methods and had a clear explanation of the sampling procedure. Compared with convenience sampling methods, this method can avoid selection bias successfully.

4. Were the measures accurately measured to reduce bias?

Before distributing questionnaires, a pilot study was applied to test the instrument. This test can improve the reliability of the questionnaire and ensure that participants have a good understanding of each question.

5. Were the data collected in a way that addressed the research issue?

Alexis (2015) adopted a questionnaire approach to collect data. This questionnaire covers all content associated with the research questions

6. Did the study have enough participants to minimize the play of chance?

There was a relatively small sample size in this design. Hence, the results cannot be directly generalized to the wider population

7. How are the results presented, and what is the main result?

$P$-value $<0.05$ was identified as a standard to assess the significance of difference in the statistics. During the process of

Yes

Yes

No

Yes

Yes

Yes

No

Yes

Can't tell

Valuable thematic analysis, sufficient data were presented in the form of 3 tables to support the findings. 
8. Was the data analysis sufficiently rigorous?

Chi-square, Kruskal-Wallis tests, and Fisher's exact test were applied for data analysis. An in-depth description of the whole analysis procedure was presented.

9. Is there a clear statement of findings?

Yes

The findings responded to the aims of this research. Moreover, based on original research questions, the findings were explicitly presented and discussed.

10. Can the results be applied to the local population?

There was a relatively small sample size in this design. Hence, the results cannot be directly generalized to the wider population.

11. How valuable is the research?

The contribution of this research was to provide quantitative data, which were missing in previous research. Importantly, the findings of this research suggested that further quantitative research should be conducted on this topic.

Valuable 